https://doi.org/10.7203/Normas.4.4685

\title{
GESTIÓN DEL LÉXICO TURÍSTICO EN PÁGINAS WEBS HOTELERAS DE PROMOCIÓN: UN EJEMPLO EN ALOJAMIENTOS DE LA COMUNIDAD BALEAR
}

\author{
TOURISTIC LEXICAL MANAGEMENT IN HOTELS'ADVERISING WEBSITES. AN \\ EXAMPLE BASED ON ACCOMODATIONS ON BALEAR ISLANDS
}

\author{
María Loreto CANTÓN RoDRÍGUEZ \\ Universidad de Almería
}

\begin{abstract}
RESUMEN:
En este trabajo se muestran las nuevas posibilidades de gestión del léxico turístico a partir del análisis de páginas webs de promoción de alojamientos turísticos con la que se ha nutrido una base de datos. Se ilustrará la influencia decisiva del canal Internet en la promoción turística y las características con sello propio del nuevo discurso turístico generado en la web 2.0. Expertos en marketing y lingüistas se adaptan al cambio radical de paradigma y de género (cibergénero, macrogénero) con productos competitivos basados en la atribución continua de nuevos significados al léxico. Finalmente se presenta un modelo de trabajo con el corpus resultante de un grupo representativo de alojamientos turísticos de la Comunidad Balear.
\end{abstract}

Palabras Clave: Léxico turístico, discurso turístico, lingüística del corpus, promoción turística.

\section{ABSTRACT:}

In this project, new posibilities of tourist lexical management are supplied by working with a well-stocked database of tourist accommodation promotional web sites. Internet decisive influence will be ilustrated on tourist promotion and on the charasteristics of a new tourist speech generated on 2.0 web. Marketing experts and linguists are getting used to this radical paradigm and genre change (cibergenre and macrogenre) with competitive products based on a continous adjudication of new lexical meanings. Finally a model is introduced as a result of working with a representative group of tourist acommodation in Balear islands.

KEY WORDS: lexical, touristic, touristic speech, corpus linguistic, touristic promotion. 


\section{INTRODUCCIÓN}

En el presente artículo ${ }^{1}$ queremos ilustrar la importancia de las investigaciones de la lingüística del corpus aplicadas al ámbito turístico, adoptando como campo de trabajo la promoción turística a partir de páginas webs de alojamientos.

Su estudio abarca varios objetivos del proyecto al que pertenece y adopta como punto de partida el análisis léxico de de corpus comparables en español, inglés y francés. Intentamos, por un lado, establecer dicho corpus de términos especializados aparecidos en las páginas webs de promoción; por otro, comparar los resultados entre las diferentes lenguas y las variedades geolectales que se derivan de cada una de ellas.

En un segundo estadio del análisis, otorgamos un valor fundamental al estudio de la traducción de estas páginas webs turísticas y subsidiariamente a los desajustes y desplazamientos producidos por una mala interpretación del léxico de especialidad.

Este enfoque parte de la necesidad de dar respuesta a profesionales de un sector que demanda una actualización continua de su imagen externa.

Ciertamente, el turismo se ha convertido en la mayor fuente de ingresos en España. En tiempos de crisis, el sector turístico ha seguido aportando cifras positivas gracias sobre todo al turismo internacional y a la recuperación del turismo nacional.

La actividad turística se desarrolla en una doble vertiente: una actividad intercultural y una actividad interlingüística. El discurso publicitario turístico producido por instituciones públicas y privadas se basa sobre todo en un amplio despliegue estratégico de persuasión basado en el estilo.

Desde sus inicios como actividad de cierta amplitud social, el turismo ha producido multitud de formatos impresos basados en una sugerente combinación de textos e imágenes.

Con el vertiginoso avance de Internet hasta llegar a su posición dominante como canal de comunicación de masas, los organismos de promoción públicos y las empresas privadas, en tanto que principales emisores del discurso publicitario, han revolucionado el concepto de venta del producto turístico. Desde sus comienzos novedosos hace poco más de un par de década, es raro hoy en día ver un negocio turístico sin sitio web. Y, más aún, la web también ha experimentado cambios radicales. La web de sus comienzos fue mucho más estática, sin posibilidades de interacción. La web 2.0 se caracteriza principalmente por las posibilidades de interacción del usuario en la propia página web o entre diferentes usuarios. De esta forma, los consumidores son proveedores de contenidos en la web que se retroalimentan. Si hace unos años la opinión de los turistas se expresaba en los libros de oro de los establecimientos, en las encuestas de opinión, de las que apenas se conocían los resultados, hoy la posibilidad de verter opiniones en la web y obtener respuesta de forma instantánea en chats, foros, blog, etc. ha provocado una reflexión sobre el cómo dar valor a un producto en el que el usuario está implicado

\footnotetext{
${ }^{1}$ Este texto se ha elaborado en el marco del Proyecto de investigación Análisis léxico y discursivo de corpus paralelos y comparables (español-inglés-francés) de páginas electrónicas de promoción turística, financiado por el Ministerio de Ciencia e Innovación. Referencia: FF2011-24712.
} 
desde el momento de su puesta en funcionamiento. Para la doctora Suau, se trata del desarrollo del nuevo concepto de Consumactor:

... se ha acuñado el nuevo término de turista 2.0 , un viajero más informado que nunca, participativo, que busca y compara y que ya no decide a partir del consejo de una agencia de viajes, sino que ha de ser persuadido directamente por el producto o servicio turístico y la forma en que se presenta en internet (Suau, 2012: 144).

Todo esto provoca la aparición de situaciones comunicativas multilingües y, con ellas, las aportaciones de la lingüística ${ }^{2}$.

Para que una web promocional turística se desarrolle en las mejores condiciones, en su diseño deberá contar con expertos informáticos, con expertos del marketing publicitario y con expertos en lingüística. En el año 1999, el Manifiesto de Cluetrain reveló una toma de conciencia de estos cambios sobrevenidos por el vertiginoso desarrollo de las TIC.

De las 95 ideas que recoge el manifiesto, la primera es la que, desde el punto de vista de la lingüística, nos sirve para reflexionar sobre la relevancia de la actuación de los consumidores en la nueva era de los mercados digitales. «Los mercados son conversaciones». Conversaciones entre vendedor y consumidor $y$, entre varios consumidores. Usar el término apropiado en el momento apropiado, servirá para dar valor al producto y para diferenciarlo de otros del mismo segmento del mercado. La tesis número 39: «La comunidad del diálogo es el mercado» ${ }^{3}$. Por tanto, ¿cómo desarrollar un discurso turístico atractivo?

\section{GÉNEROS, DISCURSOS Y LÉXICO TURÍSTICOS: REVISIÓN DE CONCEPTOS}

Los estudios acerca del discurso turístico son relativamente recientes si tenemos en cuenta que, dado el carácter híbrido de la comunicación turística, hasta ahora su clasificación no se había tenido en cuenta dentro de las denominadas lenguas de especialidad o lenguas para fines específicos.

Mourlhon-Dallies, con otros autores, acuñó el término de «lenguas profesionales», atendiendo a la demanda de un grupo de personas que se rigen por las mismas prácticas colectivas. No se trata únicamente de establecer un conjunto de necesidades

\footnotetext{
${ }^{2}$ Sophie Moirand, en el año 1990, estudió cómo las relaciones en los negocios necesitan de la linguística y de un concepto acuñado por la psicología social. Declaraba: «On envisagera surtout les "relations d'affaires" dans les domaines du commerce, de l'artisanat, de l'industrie, aires professionnelles où la communication est essentiellement interactionnelle, dans le sens où interaction, terme de la psychologie sociale signifie "volonté d'influencer l'autre" et donc modalité d'intervention langagière participant à la construction des échanges verbaux» (Moirand, 1990: 52).

${ }^{3}$ En estos casos debería entenderse el término de lingüística de formación: «la contribution des réalités langagières à la construction des identités professionnelles; le fonctionnement lexico-syntaxique de formes langagières au travail; le fonctionnement conversationnel des raisonnements et des mécanismes de prise de décision; les formes de l'écrit et les actes d'écriture au travail; la communication exolingue au travail, etc.» (Fillietaz, 2009: 1).
} 
comunicativas por parte del hablante, sino de usarlas en un contexto especializado. Según esta especialista, «Le fait est que parmi les genres usuellement catégorisés en analyse de discours, nous retenons surtout ceux centrés sur des pratiques collectives de travail, sur des habitudes» (2009: 12) ${ }^{4}$. Se trata de prácticas sociales con manifestaciones en ámbitos propios, en nuestro caso, el ámbito turístico (ferias de turismo, agencias de viaje, establecimientos hoteleros, etc.).

Las diferentes tipologías textuales que genera el discurso turístico se clasifican atendiendo a los tipos de usuarios. Dentro de los discursos científicos y técnicos, únicamente tendrían cabida aquellos ensayos o reflexiones teóricas al alcance únicamente de expertos y estudiosos de esta disciplina; aún dentro de unos receptores de expertos, pero en este caso como usuarios, se encontrarían aquellos documentos ligados a la gestión turística. Estos se clasificarían dentro de los discursos profesionales y, por último, unos documentos dirigidos al gran público clasificados dentro de lo hemos denominado la vulgarización de los discursos profesionales: folletos, catálogos, trípticos $\mathrm{y}$, sobre todo, en la actualidad, las páginas webs de promoción. Citando a Julia Sanmartín (2012: 81), «... se podrían considerar como el discurso menos especializado en el ámbito de la gestión turística, ya que se dirigen a un público lego y profano en la materia».

Para desarrollar un discurso turístico atractivo hay que tener en cuenta la creación y clasificación de los nuevos géneros que se han desarrollado de forma paralela a la aparición de las nuevas tecnologías.

La evolución de la informática y sus resultados aplicados al ámbito del turismo, han provocado un cambio también en las estrategias de marketing que los profesionales de la confección de páginas webs deben asumir.

Es evidente que el impacto de estas tecnologías ha producido cambios que afectan directamente a ese turista 2.0 del que ya hemos hablado. Las principales características que se pueden aplicar a nuestro objeto de estudio, las páginas webs de promoción, son las siguientes, atendiendo a los principios planteados por el profesor Yus Ramos (2001):

- Comunicación reticular: en una página web, cualquier creador de contenido puede también ser consumidor; de hecho, las experiencias propias pueden ser reflejadas de alguna manera en el formato y en el contenido de la página.

- Multiplicidad de formatos: ya hemos visto cómo en los cibergéneros turísticos entran a formar parte géneros más tradicionales que se han trasladado mediante el mismo formato a la web -folletos de hoteles, trípticos de monumentos, catálogos de servicios complementarios- o géneros nuevos, como los blogs o foros.

- Ubicuidad: dominio del espacio y del tiempo. En las páginas webs seleccionadas queda patente que cualquier usuario puede hacer uso de ellas en cualquier momento del día. En cuanto al espacio externo, estas pueden consultarse desde cualquier parte del mundo que ofrezca un punto Internet y,

${ }^{4}$ Calvi (2009) prefiere hablar de «lengua de especialidad» para comunicarse entre sí o dirigirse al público frente a otras denominaciones como «lenguas para fines específicos o lenguas especializadas». 
el creador dispone del espacio interno de la página para cambiar conceptos, quitar, añadir, en definitiva, rediseñar la página.

- La retroalimentación: creadores y usuarios mantienen el canal abierto a la expresión y a las sugerencias. Cualquier persona que quiera dar su opinión acerca del sitio web que visita, desde el punto de vista interno, o también de los enlaces a los que ha accedido o de su propia visita al destino o producto anunciado, podrá recibir respuesta de tipo inmediato, como en los chats de opiniones de viajeros de la página del propio establecimiento hotelero o en los enlaces a otras páginas de opiniones.

Debido al carácter interdisciplinar del discurso turístico, y siguiendo a Calvi (2010:11):

... los planteamientos basados en el concepto de género son los más adecuados para analizar las propiedades de los textos turísticos y su relación con el contexto sociocultural en el que son producidos, permitiendo así integrar la perspectiva lingüísticoformal con los enfoques pragmáticos, discursivos y sociocríticos.

La aparición de nuevos soportes y de nuevos dispositivos de comunicación, tal y como hemos observado, conlleva la creación de nuevos géneros. Algunos trabajos (Alcaraz Varó, 2000; Baider et alii, 2001; Mourlhon-Dallies, 2009; Calvi, 2010) plantean revisiones de los conceptos de género y discurso para adaptarlos a las nuevas situaciones de comunicación. Nos detendremos únicamente en el concepto de macrogénero, ya que en él se enmarcan las páginas webs de promoción hotelera. Calvi (2010: 24) resume perfectamente las características de este concepto:

... combinaciones de géneros, cuyo resultado final tiene un valor autónomo; guías, folletos y páginas webs por ejemplo contienen elementos similares pero se explayan con objetivos y estilos diferentes [...]. Los géneros, por supuesto, pueden presentarse también de forma autónoma; su inclusión en el marco de un macrogénero tiene un efecto vinculante para varias propiedades textuales (grado de informatividad, fuerza persuasiva, etc.).

En este concepto de macrogénero se unen también todas las funciones: informativa, prescriptiva, fática, etc. Las páginas webs, además de compartir estas características, se desarrollan dentro de un hipertexto navegable con todos los aspectos positivos que hemos definido más arriba.

El léxico turístico se convierte en la herramienta clave de estas páginas webs. El usuario que consulta un alojamiento a través de su página web se siente atraído, en primer lugar, por la imagen que ofrece el sitio como tal, por su navegabilidad y el acceso a la información que busca. En un segundo momento, la imagen da paso al lenguaje, al cómo se presenta la información que acompaña la imagen y qué le sugiere o le propone. La objetividad de la información hace referencia a precios, disponibilidad del alojamiento, horarios de recepción, etc., por señalar algunos, pero el cliente busca algo más que le haga diferenciar en el momento de tomar una decisión en su elección; por eso «todas las páginas webs que promocionan un destino turístico tienden a emplear 
párrafos verdaderamente plagados algunos casos de adjetivos, con el fin de persuadir, "enamorar" mediante el lenguaje al turista potencial» (Edo Marzá, 2012: 56).

La nominalización en las lenguas de especialidad es una de las características propias y, en la mayoría de las disciplinas científicas e incluso comerciales, la creación de un léxico específico exclusivo. En el caso del turismo, y debido al carácter híbrido al que ya hemos aludido, encontramos un léxico que proviene de términos del lenguaje general y de otras disciplinas. Así, distinguimos:

- Términos propios del léxico del turismo, entre los que entrarían a formar parte todos los tipos de alojamientos, actividades propias de la gestión turística, etc.

- Términos de especialidad pero pertenecientes a otras disciplinas que pueden formar parte del mundo del turismo: podemos encontrar léxico del ámbito económico, de la geografía, del arte, etc.

- Por último, términos del ámbito de lo cotidiano en donde se combinarían sustantivos con los adjetivos que reclaman la atención del potencial cliente ${ }^{5}$.

Todo esto nos ha permitido centrar nuestra investigación dentro del proyecto sobre el léxico de especialidad, teniendo en cuenta los parámetros de análisis abordados desde la teoría.

\section{Metodología de TRABAJO}

\subsection{Elección de un subcorpus}

La lingüística del corpus nos ha ofrecido la base para el análisis de datos, entendida esta según Caravedo (1999: 19):

... toda orientación que, en la formulación y en el desarrollo de su programa de investigación (comprendidos la teoría y el sistema de corroboraciones o refutaciones desprendido de la actividad analítica) depende de la observación de un conjunto de datos extraídos de la producción real de los individuos, y ordenados según criterios metodológicos diferentes pero explícitos de investigación.

La base de datos con la que obtenemos los resultados para este artículo, COMETVAL (Corpus multilingüe de Turismo de la Universidad de Valencia) se comenzó a elaborar en el año 2009 con documentos procedentes de páginas electrónicas

\footnotetext{
${ }^{5}$ Otra clasificación es la que aportó Sahar Mohlarranm-Ghinut ya en los años ochenta, en la que se unirían los dos primeros núcleos. Distingue lo que llama núcleo duro: «Le "noyau dur" caractérise et reflète le vocabulaire du domaine d'expérience. Il est composé d'un ensemble d'unités lexicales qui sont propres au domaine d'expérience et non empruntées à d'autres domaines, par exemple, agence de voyage, tour-opérateur, hôtel, agrotourisme, etc. Nous avons constaté qu'une tranche importante du "noyau dur" est formé d'après le vocabulaire économique et commercial. Ainsi, avons-nous relevé des unités telles que: "produit touristique", "acheteur du tourisme", "pays exportateur de touristes", etc.

À côté du "noyau dur" existe un ensemble d'unités lexicales qui "voyagent" entre le fond commun de la langue et le vocabulaire du tourisme et de l'hôtellerie, par exemple: cuisine, chambre, couvert, etc.».
} 
de la Comunidad Valenciana. Posteriormente, en sucesivos proyectos, el corpus se amplió recogiendo páginas webs de organismos de promoción turística institucionales y privados de todas las comunidades autónomas de España, de otros países sudamericanos, y de páginas de Francia y Canadá para la lengua francesa y de Reino Unido de EE. UU. y de Irlanda para la lengua inglesa. El objetivo es tener un corpus lo suficientemente representativo en las tres lenguas para que podamos establecer una comparativa de términos del ámbito turístico. El proyecto actual aúna, así pues, un corpus multilingüe y comparable. Este corpus nos permite obtener un glosario de términos de promoción turística de páginas institucionales y privadas de estos países.

Los investigadores han elaborado ya algunas respuestas sobre el empleo lexicográfico de estas páginas hoteleras, atendiendo a los diferentes ámbitos de la promoción hotelera: alojamientos, gestión y servicios, así como estudios referidos a las variedades geolectales y a la traducción en las distintas lenguas.

El ejemplo que presentamos en estas líneas se basa en el corpus correspondiente a las páginas webs de promoción hotelera de la Comunidad Balear, ya que se trata de uno de los destinos más visitados por los turistas extranjeros y del que nos interesaba también las traducciones a otras lenguas debido al número de visitantes extranjeros.

Según los datos obtenidos de FRONTUR, en el año 2013 las Islas Baleares fueron el segundo destino más visitado por los turistas extranjeros, con un porcentaje del $18.3 \%$, por detrás de Cataluña $(25.7 \%)$ y por delante de las Canarias $(17.5 \%)$ y de Andalucía $(13 \%)^{6}$.

La mayoría del flujo de turistas eligió los hoteles u alojamientos similares hasta un total del $64.4 \%$.

Partiendo de estos datos, es lógico plantearse un análisis de la promoción turística en estas islas con gran arraigo turístico a lo largo de los tiempos. Este hecho provoca una constante renovación de las técnicas de venta del producto turístico. Los alojamientos deben renovar constantemente sus medios de promoción. En nuestro análisis presentamos un conjunto representativo de hoteles de las principales islas de la comunidad balear.

\begin{tabular}{|l|l|}
\hline TOTAL & CATEGORÍA \\
\hline & 1 ***** \\
PALMA & $\mathbf{4}^{* * * * *}$ \\
DE MALLORCA & $\mathbf{4}^{* * * *}$ \\
& $\mathbf{1}^{* * *}$ \\
\hline & $\mathbf{3}^{* * * * *}$ \\
MENORCA & $\mathbf{3}^{* * * *}$ \\
\hline IBIZA & $\mathbf{1}^{* * *}$ \\
\hline & $\mathbf{3}^{* * * *}$ \\
\hline
\end{tabular}

Conjunto total de hoteles

${ }^{6}$ Sin embargo, aun observando los datos positivos de visitas, existe un segundo dato que nos hace dudar de la fidelización del turista por parte de los organismos privados y públicos de las Islas Baleares. Aunque se trata de datos de 2011, la encuesta de HABITUR demuestra que aunque un $61.8 \%$ de los turistas que visitan España, volverían de nuevo al mismo destino. Sin embargo, en el caso de las Islas Baleares no se produce ese efecto, por lo que es necesario una reflexión sobre la necesidad de incrementar el valor del producto turístico buscando esa fidelización del cliente. 


\subsection{Análisis y resultados}

La herramienta informática que hemos utilizado para la obtención de datos ha sido el programa Antcon 3.4.1. Este programa informático de extracción de términos y colocaciones nos permite extraer la frecuencia de términos turísticos en las páginas webs de los hoteles que tenemos registrados en la base de datos. Aun tratándose de un corpus de representación, hemos comprobado en otros trabajos, con análisis comparados dentro del corpus global, que gran parte de las unidades lexicales se repiten, diferenciándose la colocaciones, según aparezcan en uno u otro tipo de establecimiento.

Una vez introducido el corpus de trabajo, añadimos un listado de lo que se denomina palabras ruido, es decir, palabras gramaticales o funcionales ${ }^{7}$ que, en esta primera fase de obtención de términos, serían en cierta manera perjudiciales para la selección de un listado objetivo. El programa nos ofrece un listado de términos ordenados por orden alfabético de palabras o por frecuencia.

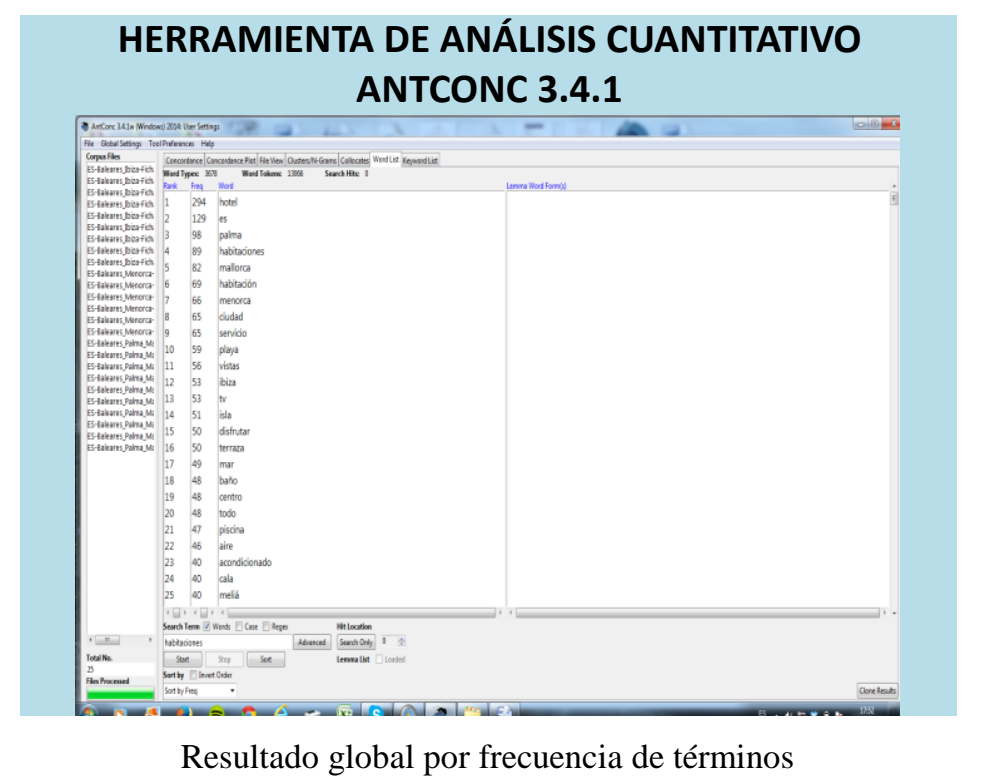

Además, el programa nos permite, accediendo a cada uno de los términos, obtener la fuente de la que proviene el término elegido, la página web del hotel, comparar las colocaciones del mismo término en este establecimiento con otro y seleccionar las colocaciones más frecuentes para, posteriormente, realizar una comparativa entre las lenguas seleccionadas dentro del proyecto.

${ }^{7} \ll \ldots$ una serie de palabras vacías de contenido y sin un valor específico para los recuentos de frecuencia o para fines terminológicos/terminográficos pero extremadamente comunes y frecuentes en cualquier tipo de texto (artículos, preposiciones...)» (Edo Marzá, 2012: 61). 


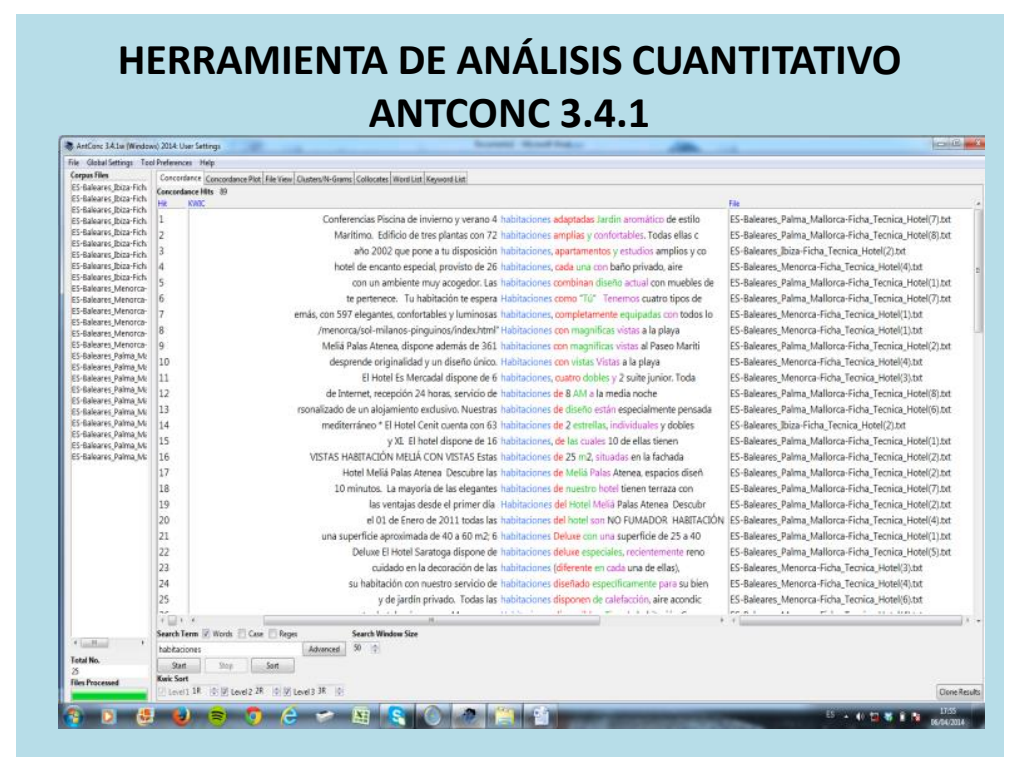

Ejemplo de colocaciones del término Habitación

Hemos obtenido el listado de los términos más frecuentes. Presentamos en el anexo II los cien sustantivos y los cien adjetivos más frecuentes.

El primer análisis llevado a cabo ha sido la clasificación de los términos más frecuentes, atendiendo al ámbito del turismo al que pertenecen. Mostramos el resultado en la siguiente tabla:

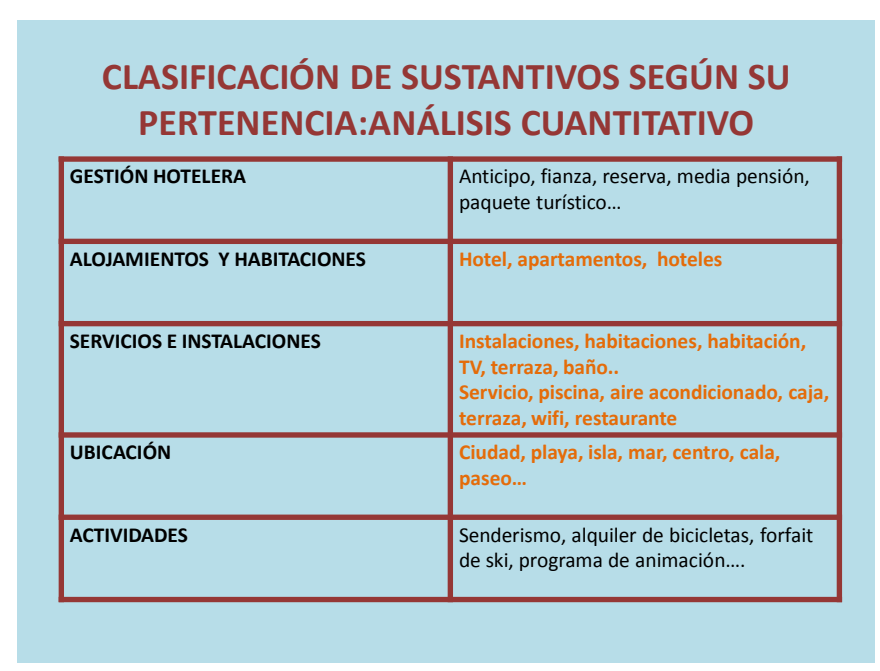

Los términos señalados en color rojo pertenecen a los que más se repiten. Es lógico pensar que el usuario necesita más información acerca del tipo de alojamiento, su ubicación y cómo se presenta ese alojamiento en cuanto a servicios e instalaciones se refiere. El potencial cliente únicamente se interesará por los otros dos campos, gestión y actividades complementarias, cuando haya optado por uno de los establecimientos gracias a los campos que considera de especial interés. 
Si pasamos ahora al análisis de sustantivos, el cliente que viaja a las Islas Baleares conoce el producto a partir del propio nombre propio de la isla: nombrar por Palma, Mallorca, Menorca o Ibiza es suficiente para vendernos el destino turístico. De ahí que estos términos aparezcan en las primeras posiciones de frecuencia:

- Palma: posición 3

- Mallorca: posición 5

- Menorca: posición 7

- Ibiza: posición 12

También es interesante destacar la influencia de las grandes cadenas turísticas que venden la imagen del producto a través de su marca de empresa. En el listado observamos la colocación de la cadena Meliá y de Tryp. Los clientes, conocedores de la trayectoria de estas grandes cadenas, se fijan y aceptan el producto por su pertenencia a ellas. Por eso, la mayoría de estas cadenas hoteleras han cambiado la configuración de sus webs: si antes vendían sus alojamientos de forma individual aun manteniéndose firmes al logo de la marca, hoy en día una mayoría opta por una web común, marca de la empresa, y el acceso a los diferentes establecimientos a partir de la web principal.

Otro grupo de unidades lexicales son las referidas al tipo de turismo que los establecimientos baleares quieren vender. En las primeras posiciones del listado obtenido encontramos los términos siguientes cercanos en lugar y en frecuencia:

$$
\begin{aligned}
& \text { - Playa (10) } \\
& \text { - Isla (14) } \\
& \text { - Mar (17) } \\
& \text { - Cala (24) }
\end{aligned}
$$

La tradición de las islas es la de promover un turismo de sol y playa. Las Islas Baleares apuestan por un turismo no estacional, europeo -esencialmente alemán- que buscan en el clima de las islas una manera de alejarse del frío de sus países de origen. Frente al cierre de establecimientos hoteleros que se puede producir en otros lugares costeros de la península, la Comunidad Balear, tanto a través de sus instituciones públicas como privadas, realizaron desde el primer momento una apuesta por este tipo de turismo, que recogen la mayoría de las webs hoteleras. Aunque el término ciudad ocupa la séptima posición, hay que ver que el contexto se refiere a la ubicación hotelera $\mathrm{y}$ no a un tipo de turismo cultural, como pudiera parecer sin las colocaciones pertinentes.

Y si desde el punto de vista de la nominalización los sustantivos funcionan dentro del repertorio tal y como se había mostrado en nuestras variables teóricas, el porcentaje de adjetivos funciona para distinguir y dar valor al contexto del establecimiento y al producto que vende.

\footnotetext{
${ }^{8}$ El número indica la posición en el listado obtenido de la herramienta Antcon.
} 
Estos términos pertenecen al ámbito de la ubicación del establecimiento. Otro grupo de unidades lexicales sustantivadas son las referidas al principal léxico del alojamiento, habitación. El campo de términos que lo acompaña para señalar las diferencias son, por orden de frecuencia, vistas, TV, terraza, baño, aire acondicionado. De hecho, el cliente pagará en función de estos servicios de la habitación. Una habitación con vistas puede elevar su precio sobre otras que no tengan vistas panorámicas o exteriores. Por lo tanto, estos términos se convierten en elementos diferenciadores que hacen único el producto.

Una de las principales características del lenguaje publicitario está en la persuasión. Atraer al cliente supone añadir valores nuevos que diferencien el producto. En los establecimientos hoteleros, la normativa indica los elementos de base con los que trabajar para ofrecer un alojamiento que conforma sus instalaciones atendiendo a lo establecido por ley. Por tanto, la diferenciación se marca en cada uno de los aspectos que lo conforman. La imagen atrae al cliente, pero a ella hay que añadir una serie de adjetivos que den valor y que singularicen ese producto.

A partir de los resultados obtenidos entre los cien adjetivos correspondientes a la base de datos analizada, podemos clasificarlos en:

- Objetivos ${ }^{9}:$ individual, completa, marítimo, mediterráneo...

- Subjetivos:

$\checkmark$ Afectivos: ideal, romántico, elegante...

$\checkmark$ Evaluativos:

- Axiológicos: Fuerte, alta, máximo...

- No axiológicos: amplia, privada, escasos...

El objetivo es marcar las diferencias entre productos que se ofrecen al consumidor a través de un mismo canal, la página web. Se hace necesaria una constante actualización de los contenidos y, en esta idea, el uso del término adecuado puede provocar un cambio de decisión por parte del consumidor.

Si además el consumidor es un turista extranjero, la persona que concibe la página web debe ser conocedora de los hábitos y costumbres de ese potencial cliente que nos va a visitar. La percepción sobre el Otro debe manifestarse también a través de las expresiones, del léxico utilizado y de una buena traducción puesta a disposición del turista extranjero. Según Bugnot (2009: 101), «En effet le touriste se construit par rapport à, et surtout en opposition à l'Autre» ${ }^{10}$.

Un análisis específico de la traducción de todas las páginas webs presentadas constituiría el objetivo de otro trabajo, pero no queremos obviar la importancia que dentro del discurso turístico tiene el trabajo de traducción de esas páginas en el uso de los términos, sustantivos y adjetivos, que con más frecuencia hemos encontrado en la

${ }^{9}$ Ejemplos extraídos del corpus según criterio de frecuencia.

10 Esta misma autora añade al discurso publicitario turístico la parte de la traducción como fundamental en la concepción y venta del producto turístico. Afirma: «L'intégration du discours touristique dans la division publicité ne se produit que dans le domaine de la recherche traductologique, linguistique et philologique» (Bugnot, 2009: 9). 
lengua de origen. La lengua meta tendrá que evitar los eufemismos y, en gran medida, los clichés, que aun vendiendo gran parte de un producto estereotipado pueden engañar al usuario de ese mismo producto.

Únicamente queremos mostrar que una conciencia lingüística se está instalando cada vez más entre los empresarios del sector turístico, como demuestran los ejemplos de las páginas elegidas. Las grandes cadenas hoteleras están a la cabeza de las traducciones de sus páginas webs a otras lenguas, pero, si nos fijamos, pequeños establecimientos más familiares han tomado conciencia de esta situación y sus páginas están traducidas, con más o menos acierto, a varias lenguas.

En la traducción, por tanto, no hay que perder los procedimientos puestos en marcha para dar valor al discurso. El funcionamiento de los adjetivos, tal y como hemos detectado en nuestro corpus, nos muestra que una buena traducción es sinónimo de una buena promoción. Sirva como ejemplo uno de los establecimientos hoteleros que hemos elegido dentro de los alojamientos baleares en sus dos versiones en español y en francés (ver anexo III).

\section{A MODO DE CONCLUSIÓN}

Los resultados expuestos en estas líneas son únicamente un ejemplo de trabajo que debe continuar en la práctica exhaustiva de los diferentes términos que definen el discurso turístico.

Por otra parte, la traducción de páginas webs hoteleras se hace imprescindible para persuadir y convencer a los diferentes tipos de clientes atendiendo a sus costumbres y usos. En este sentido, los establecimientos hoteleros deben contar con profesionales lingüísticos dentro de sus equipos de marketing.

El trabajo realizado a través de listados comparables nos ofrece la posibilidad de estudiar la influencia del léxico especializado en la promoción de productos turísticos, sobre todo de las webs que son accesibles a todo tipo de clientes y nos ayuda a aportar desde la lingüística, soluciones de mejora de esas webs y la concepción de campos de léxicos de especialidad en el campo del turismo.

El análisis que hemos planteado abre las puertas de la investigación turística a ramas que unen Internet, marketing, lingüística del corpus y traducción. El campo es amplio, y si la investigación turística ha estado callada desde el punto de vista de las lenguas para fines específicos, hoy día podemos afirmar que queda camino por recorrer y que estos caminos se abren no solo desde las diferentes ramas de las ciencias sociales, sino también de las humanidades y de la filología, gracias a la interdisciplinaridad del turismo. 


\section{REFERENCIAS BIBLIOGRÁFICAS}

AlCARAZ VARÓ, Enrique (2000): El inglés profesional y académico, Madrid, Alianza Editorial.

BAIDER, Fabienne et alii (2004): La communication touristique. Approches discursives de l'identité et l'altérité, París, l'Harmattan.

Bugnot, Marie-Ange (2009): Le discours touristique ou la réactivation du «locus amoenus», Granada, Comares.

CALvi, Maria Vittoria (2010): «Los géneros discursivos en la lengua del turismo: una propuesta de clasificación», Ibérica, 19, 9-31 [en línea]: $\langle$ http://www.aelfe.org/documents/01_19_Calvi.pdf $>$.

CARAVEDO, Rocío (1999) Lingüística del corpus. Cuestiones teóricometodológicas aplicadas al español, Salamanca, Universidad de Salamanca.

EDO MARZÁ, Nuria (2012): «Páginas web privadas e institucionales: el uso de la adjetivación en un corpus inglés-español de promoción de destinos turísticos», en Sanmartín Sáez, Julia, ed., Discurso turístico e internet, Madrid/Frankfurt, Iberoamericana Vervuert. 51-79.

FILLIETAZ, Laurent (2009): «La linguistique appliquée face aux réalités de la formation professionnelle», Bulletin suisse de linguistique appliquée, 90, 1-15.

GHINHUT, Sahar née Moharram (1985): Étude d'un vocabulaire du tourisme et de l'hôtellerie, Thèse d'état, 27 de junio de 1985, dirigida por Robert Galisson.

MoIRAND, Sophie (1990): «Décrire des discours produits dans des situations professionnelles», en Beacco, Jean-Claude y Daniel Lehmann, Publics spécifiques et communication spécialisée, París, Hachette, 52-62.

MOURLHON-DALLIES, Florence (2008): Enseigner une langue à des fins professionnelles, París, Didier.

SANMARTín SÁEZ, Julia, ed. (2012a): Discurso turístico e internet, Madrid/Frankfurt, Iberoamericana Vervuert.

SANMARTín SÁEZ, Julia (2012b): «De las normativas turísticas a las páginas electrónicas de promoción de hoteles: la clasificación hotelera desde la perspectiva lingüística» en Sanmartín Sáez, Julia, ed., Discurso turístico e internet, Madrid/Frankfurt, Iberoamericana Vervuert, 81-124.

SUAU JiMÉNEZ, Francisca (2012): «Páginas webs institucionales de promoción turística: el uso metadiscursivo interpersonal en inglés y español», en Sanmartín Sáez, Julia, ed., Discurso turístico e internet, Madrid/Frankfurt, Iberoamericana Vervuert, 125-153.

Yus RAmOS, Francisco (2001): Ciberpragmática. El uso del lenguaje en internet, Barcelona, Ariel. 
ANEXO I. Hoteles del corpus balear y lenguas de traducción de las páginas webs

\begin{tabular}{|c|c|c|}
\hline & HOTELES & LENGUAS DE LA PÁGINA WEB \\
\hline \multirow{7}{*}{ 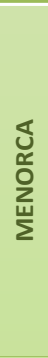 } & Playa Santandria Hotel \& Spa *** & D暗 \\
\hline & Confortel Menorca $* * *$ & r ang \\
\hline & Star San Valentín $* * * *$ & reve \\
\hline & Es Mercadal Hotel $* * * *$ & 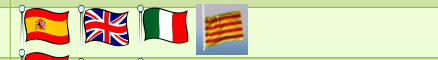 \\
\hline & Hotel Sa Barrera *** & D最口 \\
\hline & Sol Milanos Pingüinos *** & D时 E \\
\hline & Hotel Rural Sant Ignasi $* * * *$ & D敨 \\
\hline \multirow{8}{*}{$\frac{\mathbb{N}}{\underline{\underline{N}}}$} & Hotel Apartamentos El Puerto ** & O RE \\
\hline & Hotel Cenit ** & - VIVED \\
\hline & Hotel Apartamentos Lux Mar ** & N RE \\
\hline & Apartamentos Lido $* * *$ & 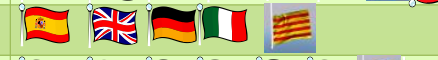 \\
\hline & Apart-Hotel Jabeque $* * *$ & 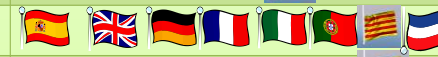 \\
\hline & Hotel Don Quijote ** & 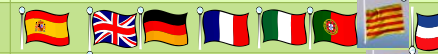 \\
\hline & Hotel Algarb $* * *$ & 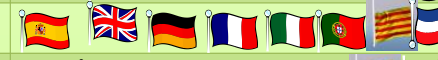 \\
\hline & Hotel Marítimo Ibiza ** & 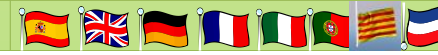 \\
\hline
\end{tabular}

\begin{tabular}{|c|c|c|}
\hline & HOTELES & LENGUAS DE LA PÁGINA WEB \\
\hline \multirow{10}{*}{ 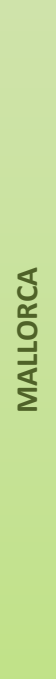 } & Boutique hotel Calatrava $* * * * *$ & 事 \\
\hline & Melià Palas Atenea $* * * *$ & 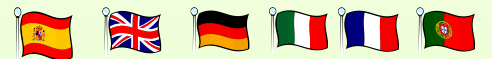 \\
\hline & Hotel Costa Azul $* * * *$ & N \\
\hline & Hotel Almudaina $* * * *$ & 盛 \\
\hline & Hotel Saratoga $* * * *$ & - E E \\
\hline & Hotel Palladium $* * *$ & i ving \\
\hline & Hotel Bosque Tryp *** & 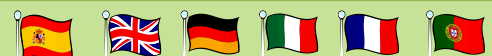 \\
\hline & Hotel Araxa *** & a vay \\
\hline & Hotel Colon Palma *** & 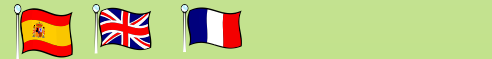 \\
\hline & Hotel Abelux ** & 具㩆 E \\
\hline
\end{tabular}


ANEXO II. Resultados obtenidos de términos por frecuencia a partir de la herramienta Antcon 3.4.1.

\begin{tabular}{|c|c|c|c|c|c|c|c|}
\hline \multicolumn{4}{|c|}{ N. N.@ ORDEN FRECUENCIA SUSTANTIVO } & \multicolumn{4}{|c|}{ N. N. ORDEN FRECUENCIA SUSTANTIVO } \\
\hline 1 & 1 & 294 & hotel & 51 & 61 & 27 & buffet \\
\hline 2 & 3 & 98 & Palma & 52 & 63 & 27 & día \\
\hline 3 & 4 & 89 & habitaciones & 53 & 66 & 27 & saratoga \\
\hline 4 & 5 & 82 & Mallorca & 54 & 67 & 26 & estancia \\
\hline 5 & 6 & 69 & habitación & 55 & 68 & 26 & $\mathrm{~km}$ \\
\hline 6 & 7 & 66 & Menorca & 56 & 71 & 25 & camas \\
\hline 7 & 8 & 65 & ciudad & 57 & 72 & 25 & petición \\
\hline 8 & 9 & 65 & servicio & 58 & 73 & 25 & reuniones \\
\hline 9 & 10 & 59 & playa & 59 & 74 & 24 & catedral \\
\hline 10 & 11 & 56 & vistas & 60 & 75 & 24 & cocina \\
\hline 11 & 12 & 53 & ibiza & 61 & 77 & 24 & minutos \\
\hline 12 & 13 & 53 & tv & 62 & 79 & 23 & aeropuerto \\
\hline 13 & 14 & 51 & isla & 63 & 80 & 23 & ambiente \\
\hline 14 & 16 & 50 & terraza & 64 & 81 & 23 & bahía \\
\hline 15 & 17 & 49 & mar & 65 & 83 & 23 & información \\
\hline 16 & 18 & 48 & baño & 66 & 88 & 22 & calefacción \\
\hline 17 & 19 & 48 & centro & 67 & 90 & 22 & internet \\
\hline 18 & 21 & 47 & piscina & 68 & 91 & 22 & reservas \\
\hline 19 & 22 & 46 & aire & 69 & 92 & 21 & barrera \\
\hline 20 & 23 & 40 & acondicionado & 70 & 93 & 21 & clientes \\
\hline 21 & 24 & 40 & cala & 71 & 94 & 21 & disposición \\
\hline 22 & 25 & 40 & meliá & 72 & 98 & 21 & playas \\
\hline 23 & 26 & 39 & apartamentos & 73 & 99 & 21 & salón \\
\hline 24 & 27 & 39 & paseo & 74 & 100 & 20 & balcón \\
\hline 25 & 29 & 38 & horas & 75 & 102 & 20 & relax \\
\hline 26 & 30 & 38 & zona & 76 & 103 & 20 & restaurantes \\
\hline 27 & 31 & 37 & hoteles & 77 & 104 & 20 & secador \\
\hline 28 & 32 & 37 & servicios & 78 & 106 & 19 & adultos \\
\hline 29 & 33 & 37 & sol & 79 & 107 & 19 & entrada \\
\hline 30 & 34 & 36 & caja & 80 & 108 & 19 & excursiones \\
\hline 31 & 36 & 36 & tryp & 81 & 114 & 19 & vacaciones \\
\hline 32 & 37 & 36 & wifi & 82 & 115 & 19 & vida \\
\hline 33 & 38 & 35 & cama & 83 & 116 & 18 & club \\
\hline 34 & 39 & 35 & restaurante & 84 & 117 & 18 & desayuno \\
\hline 35 & 41 & 34 & personas & 85 & 119 & 18 & minibar \\
\hline 36 & 42 & 34 & reserva & 86 & 120 & 18 & plasma \\
\hline 37 & 43 & 34 & spa & 87 & 122 & 18 & tipo \\
\hline 38 & 44 & 34 & teléfono & 88 & 125 & 17 & encanto \\
\hline
\end{tabular}




\begin{tabular}{|llll|llll|}
\hline 39 & 46 & 33 & puerto & 89 & 126 & 17 & estilo \\
\hline 40 & 47 & 33 & recepción & 90 & 127 & 17 & instalaciones \\
\hline 41 & 48 & 32 & bar & 91 & 129 & 17 & parte \\
\hline 42 & 52 & 29 & fotos & 92 & 131 & 17 & precio \\
\hline 43 & 53 & 29 & niños & 93 & 132 & 17 & salida \\
\hline 44 & 54 & 29 & satélite & 94 & 135 & 16 & año \\
\hline 45 & 55 & 28 & pago & 95 & 137 & 16 & equipo \\
\hline 46 & 56 & 28 & palas & 96 & 139 & 16 & masaje \\
\hline 47 & 57 & 28 & sala & 97 & 140 & 16 & noche \\
\hline 48 & 58 & 28 & vía & 98 & 142 & 16 & parada \\
\hline 49 & 59 & 27 & alquiler & 99 & 143 & 16 & planta \\
\hline 50 & 60 & 27 & atenea & 100 & 146 & 15 & acceso \\
\hline
\end{tabular}

Resultado obtenido de Antcon: frecuencia de sustantivos

\begin{tabular}{|c|c|c|c|c|c|c|c|}
\hline N.o & \multicolumn{3}{|c|}{ N. ORDEN FREC. } & \multicolumn{4}{|c|}{ N. ORDEN FREC. } \\
\hline 1 & 23 & 40 & acondicionado & 51 & 313 & 8 & cualquier \\
\hline 2 & 40 & 35 & todos & 52 & 314 & 8 & deportivo \\
\hline 3 & 45 & 33 & fuerte & 53 & 316 & 8 & diaria \\
\hline 4 & 49 & 30 & mejor & 54 & 318 & 8 & directa \\
\hline 5 & 50 & 29 & completo & 55 & 323 & 8 & exclusivas \\
\hline 6 & 64 & 27 & gran & 56 & 327 & 8 & garantizado \\
\hline 7 & 70 & 25 & cada & 57 & 331 & 8 & gratuita \\
\hline 8 & 76 & 24 & gratis & 58 & 338 & 8 & norte \\
\hline 9 & 78 & 24 & situado & 59 & 339 & 8 & público \\
\hline 10 & 84 & 23 & marítimo & 60 & 349 & 8 & tranquila \\
\hline 11 & 85 & 23 & mediterráneo & 61 & 352 & 7 & abierto \\
\hline 12 & 86 & 23 & privado & 62 & 355 & 7 & amplias \\
\hline 13 & 87 & 22 & amplia & 63 & 356 & 7 & amplio \\
\hline 14 & 89 & 22 & individual & 64 & 380 & 7 & grandes \\
\hline 15 & 97 & 21 & mejores & 65 & 383 & 7 & individuales \\
\hline 16 & 101 & 20 & doble & 66 & 384 & 7 & internacionales \\
\hline 17 & 109 & 19 & exterior & 67 & 388 & 7 & moderno \\
\hline 18 & 111 & 19 & ideal & 68 & 390 & 7 & naturales \\
\hline 19 & 118 & 18 & gratuito & 69 & 392 & 7 & náutico \\
\hline 20 & 125 & 17 & encanto & 70 & 401 & 7 & principales \\
\hline 21 & 128 & 17 & internacional & 71 & 404 & 7 & relajante \\
\hline 22 & 134 & 16 & algunas & 72 & 405 & 7 & rodeado \\
\hline 23 & 136 & 16 & equipadas & 73 & 416 & 6 & amplios \\
\hline 24 & 169 & 14 & romántico & 74 & 417 & 6 & antiguo \\
\hline 25 & 178 & 13 & dobles & 75 & 424 & 6 & buena \\
\hline 26 & 180 & 13 & elegante & 76 & 434 & 6 & confortables \\
\hline
\end{tabular}




\begin{tabular}{|c|c|c|c|c|c|c|c|}
\hline 27 & 184 & 13 & principal & 77 & 435 & 6 & conocida \\
\hline 28 & 192 & 13 & toda & 78 & 447 & 6 & espectacular \\
\hline 29 & 198 & 12 & directo & 79 & 457 & 6 & fácil \\
\hline 30 & 200 & 12 & especiales & 80 & 475 & 6 & nocturna \\
\hline 31 & 212 & 12 & primera & 81 & 479 & 6 & perfecta \\
\hline 32 & 213 & 12 & reembolsable & 82 & 480 & 6 & perfecto \\
\hline 33 & 218 & 11 & alta & 83 & 483 & 6 & pleno \\
\hline 34 & 228 & 11 & familiar & 84 & 492 & 6 & situadas \\
\hline 35 & 231 & 11 & máxima & 85 & 498 & 6 & vírgenes \\
\hline 36 & 232 & 11 & máximo & 86 & 499 & 6 & wellness \\
\hline 37 & 246 & 10 & disponibles & 87 & 500 & 6 & áreas \\
\hline 38 & 247 & 10 & escasos & 88 & 501 & 5 & aceptadas \\
\hline 39 & 248 & 10 & especial & 89 & 503 & 5 & acogedor \\
\hline 40 & 256 & 10 & luminosas & 90 & 510 & 5 & baja \\
\hline 41 & 272 & 9 & azul & 91 & 515 & 5 & buenos \\
\hline 42 & 277 & 9 & completa & 92 & 523 & 5 & completas \\
\hline 43 & 278 & 9 & comunes & 93 & 524 & 5 & confortable \\
\hline 44 & 281 & 9 & disponible & 94 & 526 & 5 & contemporáneo \\
\hline 45 & 287 & 9 & magníficas & 95 & 534 & 5 & diferentes \\
\hline 46 & 290 & 9 & privada & 96 & 535 & 5 & elegantes \\
\hline 47 & 291 & 9 & próximo & 97 & 537 & 5 & espléndido \\
\hline 48 & 297 & 9 & ubicado & 98 & 541 & 5 & exclusivo \\
\hline 49 & 300 & 9 & único & 99 & 542 & 5 & express \\
\hline 50 & 310 & 8 & comercial & 100 & 545 & 5 & famosa \\
\hline
\end{tabular}

Resultado obtenido de Antcon: frecuencia de adjetivos 
Anexo III. Ejemplo de descripción del hotel Meliá Palma en español y francés

\section{HOTEL MELIÁ PALMA ATENEA}

\section{Descripción del Hotel}

- Con espectaculares vistas panorámicas a la Bahía palmesana, la Catedral y el Paseo Marítimo, se encuentra el elegante Hotel Meliá Palas Atenea. Su estratégica ubicación a 10 minutos del aeropuerto de Mallorca y de los principales puntos históricos, culturales y turísticos de la ciudad, su excelente servicio y sus completas instalaciones hacen del Meliá Palas Ateneas el complemento perfecto para su viaje a la Isla.

- Entre su amplia oferta de instalaciones, podrás disfrutar de un moderno Business Center, completas áreas wellness y fitness, una piscina exterior, un circuito de aguas, elegante lobby y áreas comunes y magníficas terrazas desde donde podrás disfrutar de las mejores vistas de la ciudad.

\section{ELEGANTES HABITACIONES Y SUITES}

- Meliá Palas Atenea cuenta con 353 habitaciones y 8 junior suites, todas exteriores y equipadas con terraza privada donde disfrutar de un maravilloso clima mediterráneo. Ponemos a tu disposición una amplia gama de estancias que combinan por una parte el confort y por otra parte sorprendentes toques de vanguardia.

- Dotadas de 1 confortable cama king size o dos camas individuales (twin), vestidas con tejidos de alta calidad, cuentan además con una completa carta de almohadas, LCD 37", aire acondicionado con control individual frío / calor, acceso a Internet WiFi, sofá y mesita auxiliar, y confortable baño con un completo set de amenities de la marca Meliá.

\section{HOTEL MELIÁ PALMA ATENEA (versión web francés)}

\section{Description de l'hôtel}

- L'élégant hôtel Melia Palas Atenea offre des vues panoramiques spectaculaires sur la baie de Palma, la cathédrale et le front de mer, Son emplacement stratégique, à seulement 10 minutes de l'aéroport de Majorque et des principaux points historiques, culturels et touristiques de la ville, son excellent service et ses installations complètes font du Meliá Palas Ateneas le complément idéal pour votre voyage dans l'île.

- Son large éventail d'installations comprend notamment un centre d'affaires moderne, des espaces de bien-être et de remise en forme complets, une piscine extérieure, un circuit d'eau, un hall et des espaces communs élégants ainsi que de magnifiques terrasses où vous pourrez admirez les meilleures vues sur la ville

\section{CHAMBRES ET SUITES ÉLÉGANTES}

- Meliá Palas Atenea comprend 353 chambres et 8 junior suites, toutes équipées de terrasse privative où profiter de la douceur du climat méditerranéen. Nous vous proposons un grand choix d'espaces qui allient confort et modernité avec des détails surprenants.

- Équipées d'un confortable lit king size ou de deux lits individuels (twin) recouverts de textiles de qualité supérieure, carte d'oreillers, TV LCD 37", climatisation réversible avec contrôle individuel, accès à Internet Wi-Fi, canapé et table basse et salle de bain avec ensemble de produits de bain de la marque Meliá. 